IJMMS 2003:9, 557-574

PII. S0161171203007841

http://ijmms.hindawi.com

(c) Hindawi Publishing Corp.

\title{
ON THE SPECTRA OF NON-SELFADJOINT DIFFERENTIAL OPERATORS AND THEIR ADJOINTS IN DIRECT SUM SPACES
}

\author{
SOBHY EL-SAYED IBRAHIM
}

Received 20 May 2001

\begin{abstract}
The general ordinary quasidifferential expression $M_{p}$ of $n$th order, with complex coefficients and its formal adjoint $M_{p}^{+}$on any finite number of intervals $I_{p}=\left(a_{p}, b_{p}\right), p=1, \ldots, N$, are considered in the setting of the direct sums of $L_{w_{p}}^{2}\left(a_{p}, b_{p}\right)$-spaces of functions defined on each of the separate intervals. And a number of results concerning the location of the point spectra and regularity fields of general differential operators generated by such expressions are obtained.
\end{abstract}

2000 Mathematics Subject Classification: 34A05, 34L05, 47A55, 47E05.

1. Introduction. In [10, 11], Everitt considered the problem of characterizing all selfadjoint operators which can be generated by a formally symmetric Sturm-Liouville differential (quasidifferential) expression $M_{p}$, defined on a finite number of intervals $I_{p}, p=1, \ldots, N$, in the setting of direct sum spaces. In [12], the author considered the problem of the location of the point spectra and regularity fields of general ordinary quasidifferential operators on the one-interval case with one regular endpoint and the other may be regular or singular.

Our objective in this paper is to investigate the location of the point spectra and regularity fields of the operators generated by a general quas-differential expressions $M_{p}$ on any finite number of intervals $I_{p}, p=1, \ldots, N$, in the setting of direct sums of $L_{w_{p}}^{2}\left(a_{p}, b_{p}\right)$-space of functions defined on each of the separate intervals. These results extend those of the formally symmetric expression studied in $[1,2,3,15,16,17,18,19]$, and also extend those proved in $[6,12,13]$ for general case with one-interval case.

The operators involved are no longer symmetric but direct sums as

$$
T_{0}(M)=\bigoplus_{p=1}^{N} T_{0}\left(M_{P}\right), \quad T_{0}\left(M^{+}\right)=\bigoplus_{p=1}^{N} T_{0}\left(M_{P}^{+}\right),
$$

where $T_{0}\left(M_{p}\right)$ is the minimal operator generated by $M_{p}$ on $I_{p}$ and $M_{p}^{+}$is denoted by the formal adjoint of $M_{p}$ which form an adjoint pair of closed operators in $\bigoplus_{p=1}^{N} L_{w_{p}}^{2}\left(I_{p}\right)$. This fact allows us to use the abstract theory developed in [1, 2] for the operators which are regularly solvable with respect to $T_{0}\left(M_{p}\right)$ 
and $T_{0}\left(M_{p}^{+}\right)$. Such an operator $S$ satisfies $T_{0}\left(M_{p}\right) \subset S \subset\left[T_{0}\left(M_{p}^{+}\right)\right]^{*}$, and, for some $\lambda \in \mathbb{C},(S-\lambda I)$ is a Fredholm operator with zero index; this means that $S$ has the desirable Fredholm property that the equation $(S-\lambda I) u=f$ has a solution if and only if $f$ is orthogonal to the solutions of $\left(S^{*}-\bar{\lambda} I\right) v=0$, and, furthermore, the solution spaces of $(S-\lambda I) u=0$ and $\left(S^{*}-\bar{\lambda} I\right) v=0$ have the same finite dimension. This notion was originally due to Visik [20].

Throughout, we deal with a quasidifferential expression $M_{p}$ of an arbitrary order $n$ defined by a general Shin-Zettl matrix given in $[4,6,8]$, and the minimal operator $T_{0}\left(M_{p}\right)$ generated by $w_{p}^{-1} M_{p}[\cdot]$ in $L_{w_{p}}^{2}\left(I_{p}\right), p=1, \ldots, N$, where $w_{p}$ is a positive weight function on the underlying interval $I_{p}$. The endpoints of $I_{p}$ may be regular or singular.

2. Preliminaries. In this section we give some definitions and results, which will be needed later, see [3, 4, 5, 6, 9].

The domain and range of a linear operator $T$ acting in a Hilbert space $H$ are denoted by $D(T)$ and $R(T)$, respectively, and $N(T)$ denotes its null space. The nullity of $T$, written $\operatorname{nul}(T)$, is the dimension of $N(T)$ and the deficiency of $T$, written $\operatorname{def}(T)$, is the codimension of $R(T)$ in $H$; thus, if $T$ is densely defined and $R(T)$ is closed, then $\operatorname{def}(T)=\operatorname{nul}\left(T^{*}\right)$. The Fredholm domain of $T$ is (in the notation of [4]) the open subset $\triangle_{3}(T)$ of $\mathbb{C}$ consisting of those values $\lambda \in \mathbb{C}$ which are such that $T-\lambda I$ is a Fredholm operator, where $I$ is the identity operator on $H$. Thus, $\lambda \in \triangle_{3}(T)$ if and only if $(T-\lambda I)$ has closed range and finite nullity and deficiency. The index of $(T-\lambda I)$ is the number $\operatorname{ind}(T-\lambda I)=\operatorname{nul}(T-\lambda I)-\operatorname{def}(T-\lambda I)$, defined for $\lambda \in \triangle_{3}(T)$.

Two closed densely defined operators $A$ and $B$ acting in $H$ are said to form an adjoint pair if $A \subset B^{*}$ and, consequently, $B \subset A^{*}$; equivalently, $(A x, y)=$ $(x, B y)$ for all $x \in D(A)$ and $y \in D(B)$, where $(\cdot, \cdot)$ denotes the inner product on $H$.

The field of regularity $\Pi(A)$ of $A$ is the set of all $\lambda \in \mathbb{C}$ for which there exists a positive constant $K(\lambda)$ such that

$$
\|(A-\lambda I) x\| \geq K(\lambda)\|x\| \quad \forall x \in D(A),
$$

or, equivalently, on using the closed-graph theorem nul $(A-\lambda I)=0$ and $R(A-$ $\lambda I$ ) is closed.

The joint field of regularity $\Pi(A, B)$ of $A$ and $B$ is the set of $\lambda \in \mathbb{C}$ which are such that $\lambda \in \Pi(A), \bar{\lambda} \in \Pi(B)$, and both $\operatorname{def}(A-\lambda I)$ and $\operatorname{def}(B-\bar{\lambda} I)$ are finite. An adjoint pair $A$ and $B$ is said to be compatible if $\Pi(A, B) \neq \varnothing$.

DEFINITION 2.1. A closed operator $S$ in $H$ is said to be regularly solvable with respect to the compatible adjoint pair $A$ and $B$ if $A \subset S \subset B^{*}$ and $\Pi(A, B) \cap \triangle_{4}(S) \neq \varnothing$, where $\triangle_{4}(S)=\left\{\lambda: \lambda \in \triangle_{3}(S)\right.$, ind $\left.(S-\lambda I)=0\right\}$. The term "regularly solvable" comes from Visik's paper [20]. 
DEFINITION 2.2. The resolvent set $\rho(S)$ of a closed operator $S$ in $H$, consisting of the complex numbers $\lambda$ for which $(S-\lambda I)^{-1}$ exists, is defined on $H$ and is bounded. The complement of $\rho(S)$ in $\mathbb{C}$ is called the spectrum of $S$ and written $\sigma(S)$. The point spectrum $\sigma_{p}(S)$, continuous spectrum $\sigma_{c}(S)$, and residual spectrum $\sigma_{r}(S)$ are the following subsets of $\sigma(S)$ (see [3, 4]):

(a) $\sigma_{p}(S)=\{\lambda \in \sigma(S):(S-\lambda I)$ is not injective $\}$, that is, the set of eigenvalues of $S$

(b) $\sigma_{c}(S)=\{\lambda \in \sigma(S):(S-\lambda I)$ is injective, $R(S-\lambda I) \varsubsetneqq \overline{R(S-\lambda I)}=H\}$;

(c) $\sigma_{r}(S)=\{\lambda \in \sigma(S):(S-\lambda I)$ is injective, $\overline{R(S-\lambda I)} \neq H\}$.

For a closed operator $S$, we have

$$
\sigma(S)=\sigma_{p}(S) \cup \sigma_{c}(S) \cup \sigma_{r}(S)
$$

An important subset of the spectrum of a closed densely defined $S$ in $H$ is the so-called essential spectrum. The various essential spectra of $S$ are defined as in [4, Chapter II] to be the sets

$$
\sigma_{e k}(S)=\mathbb{C} \backslash \triangle_{k}(S) \quad(k=1,2,3,4,5),
$$

where $\triangle_{3}(S)$ and $\triangle_{4}(S)$ have been defined above.

The sets $\sigma_{e k}(S)$ are closed and $\sigma_{e k}(S) \subset \sigma_{e j}(S)$ if $k<j$. The inclusion is strict in general. We refer the reader to [1, 2, 3] and [4, Chapter IX] for further information about the sets $\sigma_{e k}(S)$.

3. Quasidifferential expressions. The quasidifferential expressions are defined in terms of a Shin-Zettl matrix $F_{p}$ on an interval $I_{p}$. The set $Z_{n}\left(I_{p}\right)$ of Shin-Zettl matrices on $I_{p}$ consists of $n \times n$ matrices $F_{p}=\left\{f_{r s}^{p}\right\}, 1 \leq r, s \leq n$, $p=1, \ldots, N$, whose entries are complex-valued functions on $I_{p}$ which satisfy the following conditions:

$$
\begin{gathered}
f_{r s}^{p} \in L_{\mathrm{loc}}^{1}\left(I_{p}\right) \quad(1 \leq r, s \leq n, n \geq 2), \\
f_{r, r+1}^{p} \neq 0 \quad \text { a.e on } I_{p}(1 \leq r \leq n-1), \\
f_{r s}^{p}=0 \quad \text { a.e on } I_{p}(2 \leq r+1<s \leq n), p=1, \ldots, N .
\end{gathered}
$$

For $F_{p} \in Z_{n}\left(I_{p}\right)$, the quasiderivatives associated with $F_{p}$ are defined by

$$
\begin{aligned}
y^{[0]} & :=y, \\
y^{[r]} & :=\left(f_{r, r+1}^{p}\right)^{-1}\left\{\left(y^{[r-1]}\right)^{\prime}-\sum_{s=1}^{r} f_{r s}^{p} y^{[s-1]}\right\} \quad(1 \leq r \leq n-1), \\
y^{[n]} & :=\left(y^{[n-1]}\right)^{\prime}-\sum_{s=1}^{n} f_{n s}^{p} y^{[s-1]},
\end{aligned}
$$

where the prime denotes differentiation. 
The quasidifferential expression $M_{p}$ associated with $F_{p}$ is given by

$$
M_{p}[y]:=i^{n} y^{[n]} \quad(n \geq 2),
$$

this being defined on the set

$$
V\left(M_{p}\right):=\left\{y: y^{[r-1]} \in A C_{\mathrm{loc}}\left(I_{p}\right), r=1, \ldots, n ; p=1, \ldots, N\right\},
$$

where $A C_{\text {loc }}\left(I_{p}\right)$ denotes the set of functions which are absolutely continuous on every compact subinterval of $I_{p}$.

The formal adjoint $M_{p}^{+}$of $M_{p}$ is defined by the matrix $F_{p}^{+} \in Z_{n}\left(I_{p}\right)$ given by

$$
F_{p}^{+}:=-L^{-1} F_{p}^{*} L
$$

where $F_{p}^{*}$ is the conjugate transpose of $F_{p}$ and $L_{n \times n}$ is the nonsingular $n \times n$ matrix

$$
L_{n \times n}=\left\{(-1)^{r} \delta_{r, n+1-s}\right\}, \quad(1 \leq r, s \leq n),
$$

$\delta$ being the Kronecker delta. If $F_{p}^{+}=\left(f_{r s}^{p}\right)^{+}$, then it follows that

$$
\left(f_{r s}^{p}\right)^{+}=(-1)^{r+s+1} \bar{f}_{n-s+1, n-r+1}, \quad \text { for each } r \text { and } s .
$$

The quasiderivatives associated with $F_{p}^{+}$are, therefore,

$$
\begin{aligned}
y_{+}^{[0]} & :=y, \\
y_{+}^{[r]} & :=\left(\bar{f}_{n-r, n-r+1}\right)^{-1}\left\{\left(y_{+}^{[r-1]}\right)^{\prime}-\sum_{s=1}^{r}{\overline{f^{p}}}_{n-s+1, n-r+1} y_{+}^{[s-1]}\right\}, \quad(1 \leq r \leq n-1), \\
y_{+}^{[n]} & :=\left(y_{+}^{[n-1]}\right)^{\prime}-\sum_{s=1}^{n} \bar{f}_{n-s+1,1} y_{+}^{[s-1]}, \\
M_{p}^{+}[y] & :=i^{n} y_{+}^{[n]}, \quad p=1, \ldots, N, \forall y \in V\left(M_{p}^{+}\right), \\
V\left(M_{p}^{+}\right) & :=\left\{y: y_{+}^{[r-1]} \in A C_{\mathrm{loc}}\left(I_{p}\right), r=1, \ldots, n ; p=1, \ldots, N\right\} .
\end{aligned}
$$

Note that $\left(F_{p}^{+}\right)^{+}=F_{p}$ and so $\left(M_{p}^{+}\right)^{+}=M_{p}$. We refer to $[6,12,13,14,21]$ for a full account of the above and subsequent results on quasidifferential expressions.

Let the interval $I_{p}$ have endpoints $a_{p}$ and $b_{p}\left(-\infty \leq a_{p}<b_{p} \leq \infty\right)$, and let $w_{p}: I_{p} \rightarrow \mathbb{R}$ be a nonnegative weight function with $w_{p} \in L_{\mathrm{loc}}^{1}\left(I_{p}\right)$ and $w_{p}\left(I_{p}\right)>0$ (for almost all $x \in I_{p}$ ). Then, $H_{p}=L_{w_{p}}^{2}\left(I_{p}\right)$ denotes the Hilbert function space of equivalence classes of Lebesgue measurable functions such that $\int_{I_{p}} w_{p}|f|^{2}<$ $\infty$; the inner product is defined by

$$
(f, g)_{p}:=\int_{I_{p}} w_{p}(x) f(x) \overline{g(x)} d x \quad\left(f, g \in L_{w_{p}}^{2}\left(I_{p}\right), p=1, \ldots, N\right) .
$$


The equation

$$
M_{p}[u]-\lambda w_{p} u=0 \quad(\lambda \in \mathbb{C}) \text { on } I_{p}
$$

is said to be regular at the left endpoint $a_{p} \in \mathbb{R}$ if, for all $X \in\left(a_{p}, b_{p}\right)$,

$$
a_{p} \in \mathbb{R}, \quad w_{p}, f_{r s}^{p} \in L^{1}\left[a_{p}, X\right], \quad(r, s=1, \ldots, n ; p=1, \ldots, N) .
$$

Otherwise, (3.10) is said to be singular at $a_{p}$. If (3.10) is regular at both endpoints, then it is said to be regular; in this case we have

$$
a_{p}, b_{p} \in \mathbb{R}, \quad w_{p}, f_{r s}^{p} \in L^{1}\left(a_{p}, b_{p}\right), \quad(r, s=1, \ldots, n ; p=1, \ldots, N)
$$

We will be concerned with the case when $a_{p}$ is a regular endpoint of (3.10), the endpoint $b_{p}$ being allowed to be either regular or singular. Note that, in view of (3.7), an endpoint of $I_{p}$ is regular for (3.10) if and only if it is regular for the equation

$$
M_{p}^{+}[v]-\bar{\lambda} w_{p} v=0 \quad(\lambda \in \mathbb{C}) \text { on } I_{p}, p=1, \ldots, N
$$

Note that, at regular endpoint $a_{p}$, say, $u^{[r-1]}\left(a_{p}\right)\left(v_{+}^{[r-1]}\left(a_{p}\right)\right), r=1, \ldots, n$, is defined for all $u \in V\left(M_{p}\right)\left(v \in V\left(M_{p}^{+}\right)\right)$. Set

$$
\begin{aligned}
& D\left(M_{p}\right):=\left\{u: u \in V\left(M_{p}\right), u, w_{p}^{-1} M_{p}[u] \in L_{w_{p}}^{2}\left(a_{p}, b_{p}\right)\right\}, \\
& D\left(M_{p}^{+}\right):=\left\{v: v \in V\left(M_{p}^{+}\right), v, w_{p}^{-1} M_{p}^{+}[v] \in L_{w_{p}}^{2}\left(a_{p}, b_{p}\right)\right\}, \quad p=1, \ldots, N .
\end{aligned}
$$

The subspaces $D\left(M_{p}\right)$ and $D\left(M_{p}^{+}\right)$of $L_{w_{p}}^{2}\left(a_{p}, b_{p}\right)$ are domains of the socalled maximal operators $T\left(M_{p}\right)$ and $T\left(M_{p}^{+}\right)$, respectively, defined by

$$
\begin{array}{ll}
T\left(M_{p}\right) u:=w_{p}^{-1} M_{p}[u] & \left(u \in D\left(M_{p}\right)\right), \\
T\left(M_{p}^{+}\right) v:=w_{p}^{-1} M_{p}^{+}[v] & \left(v \in D\left(M_{p}^{+}\right)\right) .
\end{array}
$$

For the regular problem, the minimal operators $T_{0}\left(M_{p}\right)$ and $T_{0}\left(M_{p}^{+}\right), p=$ $1, \ldots, N$, are the restrictions of $w_{p}^{-1} M_{p}[u]$ and $w_{p}^{-1} M_{p}^{+}[v]$ to the subspaces

$$
\begin{array}{r}
D_{0}\left(M_{p}\right):=\left\{u: u \in D\left(M_{p}\right), u^{[r-1]}\left(a_{p}\right)=u^{[r-1]}\left(b_{p}\right)=0, r=1, \ldots, n\right\}, \\
D_{0}\left(M_{p}^{+}\right):=\left\{v: v \in D\left(M_{p}^{+}\right), v_{+}^{[r-1]}\left(a_{p}\right)=v_{+}^{[r-1]}\left(b_{p}\right)=0, r=1, \ldots, n\right\}, \\
p=1, \ldots, N,
\end{array}
$$

respectively. The subspaces $D_{0}\left(M_{p}\right)$ and $D_{0}\left(M_{p}^{+}\right)$are dense in $L_{w_{p}}^{2}\left(a_{p}, b_{p}\right)$, and $T_{0}\left(M_{p}\right)$ and $T_{0}\left(M_{p}^{+}\right)$are closed operators (see $[4,6,13]$ and [21, Section 3]). 
In the singular problem, we first introduce the operators $T_{0}^{\prime}\left(M_{p}\right)$ and $T_{0}^{\prime}\left(M_{p}^{+}\right)$, $T_{0}^{\prime}\left(M_{p}\right)$ being the restriction of $w_{p}^{-1} M_{p}[\cdot]$, to the subspace

$$
D_{0}^{\prime}\left(M_{p}\right):=\left\{u: u \in D\left(M_{p}\right), \operatorname{supp} u \subset\left(a_{p}, b_{p}\right)\right\}, \quad p=1, \ldots, N,
$$

and with $T_{0}^{\prime}\left(M_{p}^{+}\right)$defined similarly. These operators are densely defined and closable in $L_{w_{p}}^{2}\left(a_{p}, b_{p}\right)$; and we defined the minimal operators $T_{0}\left(M_{p}\right)$ and $T_{0}\left(M_{p}^{+}\right)$to be their respective closures (see [4, 6] and [21, Section 5]). We denote the domains of $T_{0}\left(M_{p}\right)$ and $T_{0}\left(M_{p}^{+}\right)$by $D_{0}\left(M_{p}\right)$ and $D_{0}\left(M_{p}^{+}\right)$, respectively. It can be shown that

$$
\begin{aligned}
& u \in D_{0}\left(M_{p}\right) \Longrightarrow u^{[r-1]}\left(a_{p}\right)=0 \quad(r=1, \ldots, n ; p=1, \ldots, N) \\
& v \in D_{0}\left(M_{p}^{+}\right) \Longrightarrow v_{+}^{[r-1]}\left(a_{p}\right)=0 \quad(r=1, \ldots, n ; p=1, \ldots, N)
\end{aligned}
$$

because we are assuming that $a_{p}$ is a regular endpoint. Moreover, in both regular and singular problems, we have

$$
T_{0}^{*}\left(M_{p}\right)=T\left(M_{p}^{+}\right), \quad T^{*}\left(M_{p}\right)=T_{0}\left(M_{p}^{+}\right), \quad p=1, \ldots, N
$$

see [21, Section 5] in the case when $M_{p}=M_{p}^{+}$and compare with the treatment in [4, Section III.10.3] and [6] in the general case.

In the case of two singular endpoints, the problem on $\left(a_{p}, b_{p}\right)$ is effectively reduced to the problems with one singular endpoint on the intervals $\left(a_{p}, c_{p}\right]$ and $\left[c_{p}, b_{p}\right)$, where $c_{p} \in\left(a_{p}, b_{p}\right)$. We denote by $T\left(M_{p} ; a_{p}\right)$ and $T\left(M_{p} ; b_{p}\right)$ the maximal operators with domains $D\left(M_{p} ; a_{p}\right)$ and $D\left(M_{p} ; b_{p}\right)$, and denote by $T_{0}\left(M_{p} ; a_{p}\right)$ and $T_{0}\left(M_{p} ; b_{p}\right)$ the closures of the operators $T_{0}^{\prime}\left(M_{p} ; a_{p}\right)$ and $T_{0}^{\prime}\left(M_{p} ; b_{p}\right)$ defined in (3.17) on the intervals $\left(a_{p}, c_{p}\right]$ and $\left[c_{p}, b_{p}\right)$, respectively, see $[4,9,13,14,15,16]$.

Let $\tilde{T}_{0}^{\prime}\left(M_{p}\right), p=1, \ldots, N$, be the orthogonal sum as

$$
\tilde{T}_{0}^{\prime}\left(M_{p}\right)=T_{0}^{\prime}\left(M_{p} ; a_{p}\right) \bigoplus T_{0}^{\prime}\left(M_{p} ; b_{p}\right)
$$

in

$$
L_{w_{p}}^{2}\left(a_{p}, b_{p}\right)=L_{w_{p}}^{2}\left(a_{p}, c_{p}\right) \bigoplus L_{w_{p}}^{2}\left(c_{p}, b_{p}\right)
$$

$\tilde{T}_{0}^{\prime}\left(M_{p}\right)$ is densely defined and closable in $L_{w_{p}}^{2}\left(a_{p}, b_{p}\right)$ and its closure is given by

$$
\tilde{T}_{0}\left(M_{p}\right)=T_{0}\left(M_{p} ; a_{p}\right) \bigoplus T_{0}\left(M_{p} ; b_{p}\right), \quad p=1, \ldots, N
$$


Also,

$$
\begin{aligned}
& \operatorname{nul}\left[\tilde{T}_{0}\left(M_{p}\right)-\lambda I\right]=\operatorname{nul}\left[T_{0}\left(M_{p} ; a_{p}\right)-\lambda I\right]+\operatorname{nul}\left[T_{0}\left(M_{p} ; b_{p}\right)-\lambda I\right], \\
& \operatorname{def}\left[\tilde{T}_{0}\left(M_{p}\right)-\lambda I\right]=\operatorname{def}\left[T_{0}\left(M_{p} ; a_{p}\right)-\lambda I\right]+\operatorname{def}\left[T_{0}\left(M_{p} ; b_{p}\right)-\lambda I\right],
\end{aligned}
$$

and $R\left[\tilde{T}_{0}\left(M_{p}\right)-\lambda I\right]$ is closed if and only if $R\left[T_{0}\left(M_{p} ; a_{p}\right)-\lambda I\right]$ and $R\left[T_{0}\left(M_{p} ; b_{p}\right)\right.$ $-\lambda I]$ are both closed. These results imply, in particular, that

$$
\Pi\left[\tilde{T}_{0}\left(M_{p}\right)\right]=\Pi\left[T_{0}\left(M_{p} ; a_{p}\right)\right] \cap \Pi\left[T_{0}\left(M_{p} ; b_{p}\right)\right], \quad p=1, \ldots, N
$$

We refer to [4, Section 3.10.4] and [13, 14] for more details.

REMARK 3.1. If $S_{p}^{a_{p}}$ is a regularly solvable extension of $T_{0}\left(M_{p} ; a_{p}\right)$ and $S_{p}^{b_{p}}$ is a regularly solvable extension of $T_{0}\left(M_{p} ; b_{p}\right)$, then $S=S_{p}^{a_{p}} \oplus S_{p}^{b_{p}}$ is a regularly solvable extension of $\tilde{T}_{0}\left(M_{p}\right), p=1, \ldots, N$. We refer to [4, Section 3.10.4] and $[13,14]$ for more details.

Next, we state the following results; the proof is similar to that in [4, Section 3.10.4] and [13, 14].

THEOREM 3.2. Let

$$
\begin{gathered}
\tilde{T}_{0}\left(M_{p}\right) \subset T_{0}\left(M_{p}\right), \quad T\left(M_{p}\right) \subset T\left(M_{p} ; a_{p}\right) \bigoplus T\left(M_{p} ; b_{p}\right), \\
\operatorname{dim}\left\{D\left[T_{0}\left(M_{p}\right)\right] / D\left[\tilde{T}_{0}\left(M_{0}\right)\right]\right\}=n, \quad p=1, \ldots, N .
\end{gathered}
$$

If $\lambda \in \Pi\left[\tilde{T}_{0}\left(M_{p}\right)\right] \cap \triangle_{3}\left[T_{0}\left(M_{p}\right)-\lambda I\right]$, then

$$
\text { ind }\left[T_{0}\left(M_{p}\right)-\lambda I\right]=n-\operatorname{def}\left[T_{0}\left(M_{p} ; a_{p}\right)-\lambda I\right]-\operatorname{def}\left[T_{0}\left(M_{p} ; b_{p}\right)-\lambda I\right] \text {, }
$$

and, in particular, if $\lambda \in \Pi\left[T_{0}\left(M_{p}\right)\right]$,

$$
\operatorname{def}\left[T_{0}\left(M_{p}\right)-\lambda I\right]=\operatorname{def}\left[T_{0}\left(M_{p} ; a_{p}\right)-\lambda I\right]+\operatorname{def}\left[T_{0}\left(M_{p} ; b_{p}\right)-\lambda I\right]-n
$$

REMARK 3.3. It can be shown that

$$
\begin{aligned}
& D\left[\tilde{T}_{0}\left(M_{p}\right)\right]=\left\{u: u \in D\left[T_{0}\left(M_{p}\right)\right], u^{[r-1]}\left(c_{p}\right)=0, r=1, \ldots, n\right\}, \\
& D\left[\tilde{T}_{0}\left(M_{p}^{+}\right)\right]=\left\{v: v \in D\left[T_{0}\left(M_{p}^{+}\right)\right], v_{+}^{[r-1]}\left(c_{p}\right)=0, r=1, \ldots, n\right\}, \quad p=1, \ldots, N ;
\end{aligned}
$$

see [4, Section 3.10.4]. 
LEMMA 3.4. For $\lambda \in \Pi\left[T_{0}\left(M_{p}\right), T_{0}\left(M_{p}^{+}\right)\right], \operatorname{def}\left[T_{0}\left(M_{p}\right)-\lambda I\right]+\operatorname{def}\left[T_{0}\left(M_{p}^{+}\right)-\right.$ $\bar{\lambda} I]$ is constant and

$$
0 \leq \operatorname{def}\left[T_{0}\left(M_{p}\right)-\lambda I\right]+\operatorname{def}\left[T_{0}\left(M_{p}^{+}\right)-\bar{\lambda} I\right] \leq 2 n, \quad p=1, \ldots, N
$$

In the problem with one singular endpoint,

$$
n \leq \operatorname{def}\left[T_{0}\left(M_{p}\right)-\lambda I\right]+\operatorname{def}\left[T_{0}\left(M_{p}^{+}\right)-\bar{\lambda} I\right] \leq 2 n \quad \forall \lambda \in \Pi\left[T_{0}\left(M_{p}\right), T_{0}\left(M_{p}^{+}\right)\right]
$$

In the regular problem,

$$
\operatorname{def}\left[T_{0}\left(M_{p}\right)-\lambda I\right]+\operatorname{def}\left[T_{0}\left(M_{p}^{+}\right)-\bar{\lambda} I\right]=2 n \quad \forall \lambda \in \Pi\left[T_{0}\left(M_{p}\right), T_{0}\left(M_{p}^{+}\right)\right]
$$

Proof. See [4, 6] and [14, Lemma 2.4].

Let $H$ be the direct sum

$$
H=\bigoplus_{p=1}^{N} H_{p}=\bigoplus_{p=1}^{N} L_{w_{p}}^{2}\left(a_{p}, b_{p}\right) .
$$

The elements of $H$ will be denoted by $\tilde{f}=\left\{f_{1}, \ldots, f_{N}\right\}$ with $f_{1} \in H_{1}, \ldots, f_{N} \in H_{N}$.

REMARK 3.5. When $I_{i} \cap I_{j}=\varnothing, i \neq j$, and $i, j=1, \ldots, N$, the direct sum space $\bigoplus_{p=1}^{N} L_{w_{p}}^{2}\left(a_{p}, b_{p}\right)$ can be naturally identified with the space $L_{w}^{2}\left(\cup_{p=1}^{N} I_{p}\right)$, where $w_{p}=w$ on $I_{p}, p=1, \ldots, N$. This remark is of significance when $\cup_{p=1}^{N} I_{p}$ is taken as a single interval, see $[10,11]$.

We now establish by $[4,10,14]$ some further notations

$$
\begin{aligned}
& D_{0}(M)=\bigoplus_{p=1}^{N} D_{0}\left(M_{p}\right), \quad D(M)=\bigoplus_{p=1}^{N} D\left(M_{p}\right), \\
& D_{0}\left(M^{+}\right)=\bigoplus_{p=1}^{N} D_{0}\left(M_{p}^{+}\right), \quad D\left(M^{+}\right)=\bigoplus_{p=1}^{N} D\left(M_{p}^{+}\right), \\
& T_{0}(M) f:=\left\{T_{0}\left(M_{1}\right) f_{1}, \ldots, T_{0}\left(M_{N}\right) f_{N}\right\} ; \quad f_{1} \in D_{0}\left(M_{1}\right), \ldots, f_{N} \in D_{0}\left(M_{N}\right), \\
& T_{0}\left(M^{+}\right) g:=\left\{T_{0}\left(M_{1}^{+}\right) g_{1}, \ldots, T_{0}\left(M_{N}^{+}\right) g_{N}\right\} ; \quad g_{1} \in D_{0}\left(M_{1}^{+}\right), \ldots, g_{N} \in D_{0}\left(M_{N}^{+}\right) .
\end{aligned}
$$

Also,

$$
\begin{array}{cl}
T(M) f:=\left\{T\left(M_{1}\right) f_{1}, \ldots, T\left(M_{N}\right) f_{N}\right\} ; & f_{1} \in D\left(M_{1}\right), \ldots, f_{N} \in D\left(M_{N}\right), \\
T\left(M^{+}\right) g:=\left\{T\left(M_{1}^{+}\right) g_{1}, \ldots, T\left(M_{N}^{+}\right) g_{N}\right\} ; \quad g_{1} \in D\left(M_{1}^{+}\right), \ldots, g_{N} \in D\left(M_{N}^{+}\right) .
\end{array}
$$

We summarize a few additional properties of $T_{0}(M)$ in the form of a lemma. 
LEMMA 3.6. (a) The direct sums of $\left[T_{0}(M)\right]^{*}$ and $\left[T_{0}\left(M^{+}\right)\right]^{*}$ are given by

$$
\begin{aligned}
{\left[T_{0}(M)\right]^{*} } & =\bigoplus_{p=1}^{N}\left[T_{0}\left(M_{p}\right)\right]^{*}=\bigoplus_{p=1}^{N}\left[T\left(M_{p}^{+}\right)\right], \\
{\left[T_{0}\left(M^{+}\right)\right]^{*} } & =\bigoplus_{p=1}^{N}\left[T_{0}\left(M_{p}^{+}\right)\right]^{*}=\bigoplus_{p=1}^{N}\left[T\left(M_{p}\right)\right] .
\end{aligned}
$$

In particular,

$$
\begin{gathered}
D\left[T_{0}(M)\right]^{*}=D\left[T\left(M^{+}\right)\right]=\bigoplus_{p=1}^{N} D\left[T\left(M_{p}^{+}\right)\right], \\
D\left[T_{0}\left(M^{+}\right)\right]^{*}=D[T(M)]=\bigoplus_{p=1}^{N} D\left[T\left(M_{p}\right)\right],
\end{gathered}
$$

(b) The nullities of $T_{0}(M)$ and $T_{0}\left(M^{+}\right)$are give by

$$
\begin{aligned}
\operatorname{nul}\left[T_{0}(M)-\lambda I\right] & =\sum_{p=1}^{N} \operatorname{nul}\left[T_{0}\left(M_{p}\right)-\lambda I\right], \\
\operatorname{nul}\left[T_{0}\left(M^{+}\right)-\bar{\lambda} I\right] & =\sum_{p=1}^{N} \operatorname{nul}\left[T_{0}\left(M_{p}^{+}\right)-\bar{\lambda} I\right] .
\end{aligned}
$$

(c) The deficiency indices of $T_{0}(M)$ are given by

$$
\begin{gathered}
\operatorname{def}\left[T_{0}(M)-\lambda I\right]=\sum_{p=1}^{N} \operatorname{def}\left[T_{0}\left(M_{p}\right)-\lambda I\right] \quad \forall \lambda \in \Pi\left[T_{0}\left(M_{p}\right), T_{0}\left(M_{p}^{+}\right)\right], \\
\operatorname{def}\left[T_{0}\left(M^{+}\right)-\bar{\lambda} I\right]=\sum_{p=1}^{N} \operatorname{def}\left[T_{0}\left(M_{p}^{+}\right)-\bar{\lambda} I\right] \quad \forall \lambda \in \Pi\left[T_{0}\left(M_{p}\right), T_{0}\left(M_{p}^{+}\right)\right] .
\end{gathered}
$$

Proof. Part (a) follows immediately from the definition of $T_{0}(M)$ and from the general definition of an adjoint operator. The other parts are either direct consequences of part (a) or follows immediately from the definitions.

LEMMA 3.7. Let $T_{0}(M)=\bigoplus_{p=1}^{N} T_{0}\left(M_{p}\right)$ be a closed densely defined operator on $H$. Then

$$
\Pi\left[T_{0}(M)\right]=\cap_{p=1}^{N} \Pi\left[T_{0}\left(M_{p}\right)\right]
$$

Proof. The proof follows from Lemma 3.4 and since $R\left[T_{0}\left(M_{p}\right)-\lambda I\right]$ is closed if and only if $R\left[T_{0}\left(M_{p}\right)-\lambda I\right], p=1, \ldots, N$, are closed.

LEMMA 3.8. If $S_{p}, p=1, \ldots, N$, are regularly solvable with respect to $T_{0}\left(M_{p}\right)$ and $T_{0}\left(M_{p}^{+}\right)$, then $S=\bigoplus_{p=1}^{N} S_{p}$ is regularly solvable with respect to $T_{0}(M)$ and $T_{0}\left(M^{+}\right)$. 
Proof. The proof follows from Lemmas 3.4 and 3.6.

REMARK 3.9. Let $S=\bigoplus_{j=1}^{N} S_{j}$ be an arbitrary closed operator on $H$. Since $\lambda \in \rho(S)$ if and only if $\operatorname{nul}(S-\lambda I)=\operatorname{def}(S-\lambda I)=0$, see [3, Theorem 1.3.2], we have $\rho(S)=\cap_{j=1}^{N} \rho\left(S_{j}\right)$ and hence,

$$
\sigma(S)=\cup_{j=1}^{N} \sigma\left(S_{j}\right), \quad \sigma_{p}(S)=\cup_{j=1}^{N} \sigma_{p}\left(S_{j}\right), \quad \sigma_{r}(S)=\cup_{j=1}^{N} \sigma_{r}\left(S_{j}\right)
$$

Also,

$$
\sigma_{e k}(S)=\cup_{j=1}^{N} \sigma_{e k}\left(S_{j}\right), \quad k=2,3
$$

We refer to [4, Chapter 9] for more details.

THEOREM 3.10. Suppose that $f \in L_{\mathrm{loc}}^{1}\left(I_{p}\right)$ and suppose that conditions (3.1) are satisfied. Then, given any complex numbers $c_{j} \in \mathbb{C}, j=0,1, \ldots, n-1$, and $x_{0} \in\left(a_{p}, b_{p}\right)$, there exists a unique solution of $M_{p}\left[\phi_{p}\right]=w \phi_{p} f$ in $\left(a_{p}, b_{p}\right)$ which satisfies

$$
\phi_{p}^{[j]}\left(x_{0}\right)=c_{j} \quad(j=0,1, \ldots, n-1 ; p=1, \ldots, N) .
$$

Proof. See [1, 2, 4] and [16, Theorem 16.2.2].

THEOREM 3.11 (cf. [4] and [16, Lemma 5.17.1]). Let $M_{p}$ be a regular quasidifferential expression of order $n$ on the closed interval $\left[a_{p}, b_{p}\right]$. For $f \in$ $L_{w}^{2}\left(a_{p}, b_{p}\right)$, the equation $M_{p}[u]=w f$ has a solution $\phi_{p} \in V\left(M_{p}\right)$ satisfying

$$
\phi_{p}^{[j]}\left(a_{p}\right)=\phi_{p}^{[j]}\left(b_{p}\right)=0, \quad(j=0,1, \ldots, n-1 ; p=1, \ldots, N),
$$

if and only if $f$ is orthogonal in $L_{w}^{2}\left(a_{p}, b_{p}\right)$ to the solution space of $M_{p}^{+}\left[\phi_{p}\right]=0$, that is,

$$
R\left[T_{0}\left(M_{p}\right)-\lambda I\right]=N\left[T\left(M_{p}^{+}\right)-\bar{\lambda} I\right]^{\perp}, \quad p=1, \ldots, N
$$

COROLlARY 3.12 (cf. [16, Section 5.17.3]). As a result from Theorem 3.11, we have

$$
R\left[T_{0}\left(M_{p}\right)-\lambda I\right]^{\perp}=N\left[T\left(M_{p}^{+}\right)-\bar{\lambda} I\right], \quad p=1, \ldots, N
$$

LEMMA 3.13 (cf. [4, Lemma IX.9.1]). If $I_{p}=\left[a_{p}, b_{p}\right]$, with $-\infty<a_{p}<b_{p}<\infty$, $p=1, \ldots, N$, then, for any $\lambda \in \mathbb{C}$, the operator $\left[T_{0}\left(M_{p}\right)-\lambda I\right], p=1, \ldots, N$, has 
closed range, zero nullity, and deficiency $n$. Hence,

$$
\sigma_{e k}\left[T_{0}\left(M_{p}\right)\right]= \begin{cases}\varnothing & (k=1,2,3), \\ \mathbb{C} & (k=4,5),\end{cases}
$$

where, $p=1, \ldots, N$.

4. The spectra of operators in direct sum spaces. In this section, we consider our interval to be $I=[a, b)$. We denote by $T(M)$ and $T_{0}(M)$ the maximal and minimal operators defined on the interval $I$. Also, we deal with the various components of the spectra of $T_{0}(M)$ and $T_{0}\left(M^{+}\right)$as the direct sum of differential operators $T_{0}\left(M_{p}\right)$ and $T_{0}\left(M_{p}^{+}\right), p=1, \ldots, N$.

LEMmA 4.1. Let $T_{0}(M)=\bigoplus_{j=1}^{N} T_{0}\left(M_{j}\right)$ and $T_{0}\left(M^{+}\right)=\bigoplus_{j=1}^{N} T_{0}\left(M_{j}^{+}\right)$, then the point spectra $\sigma_{p}\left[T_{0}(M)\right]$ and $\sigma_{p}\left[T_{0}\left(M^{+}\right)\right]$of $T_{0}(M)$ and $T_{0}\left(M^{+}\right)$are empty.

Proof. Let $\lambda \in \sigma_{p}\left[T_{0}\left(M_{j}\right)\right]$. Then, there exists a nonzero element $\phi_{j} \in$ $D_{0}\left(M_{j}\right), j=1, \ldots, N$, such that

$$
\left[T_{0}\left(M_{j}\right)-\lambda I\right] \phi_{j}=0, \quad j=1, \ldots, N \text {. }
$$

In particular, this gives that

$$
\begin{gathered}
{\left[T_{0}\left(M_{j}\right)\right] \phi_{j}=\lambda w \phi_{j},} \\
\phi_{j}^{[r]}\left(a_{j}\right)=\phi_{j}^{[r]}\left(b_{j}\right)=0, \quad(r=0,1, \ldots, n-1 ; j=1, \ldots, N) .
\end{gathered}
$$

From Theorem 3.10, it follows that $\phi_{j}=0$ and hence, $\sigma_{p}\left[T_{0}\left(M_{j}\right)\right]=\varnothing, j=$ $1, \ldots, N$. Similarly,

$$
\sigma_{p}\left[T_{0}\left(M_{j}^{+}\right)\right]=\varnothing, \quad j=1, \ldots, N
$$

Therefore, by (3.40), we have

$$
\begin{aligned}
\sigma_{p}\left[T_{0}(M)\right] & =\cup_{j=1}^{N} \sigma_{p}\left[T_{0}\left(M_{j}\right)\right]=\varnothing, \\
\sigma_{p}\left[T_{0}\left(M^{+}\right)\right] & =\cup_{j=1}^{N} \sigma_{p}\left[T_{0}\left(M_{j}^{+}\right)\right]=\varnothing .
\end{aligned}
$$

THEOREM 4.2. Let $T_{0}(M)=\bigoplus_{j=1}^{N} T_{0}\left(M_{j}\right)$ and $T_{0}\left(M^{+}\right)=\bigoplus_{j=1}^{N} T_{0}\left(M_{j}^{+}\right)$, then

(i) $\rho\left[T_{0}(M)\right]=\rho\left[T_{0}\left(M^{+}\right)\right]=\varnothing$,

(ii) $\sigma_{c}\left[T_{0}(M)\right]=\sigma_{c}\left[T_{0}\left(M^{+}\right)\right]=\varnothing$,

(iii) $\sigma\left[T_{0}(M)\right]=\sigma\left[T_{0}\left(M^{+}\right)\right]=\mathbb{C}$ and $\sigma_{r}\left[T_{0}(M)\right]=\sigma_{r}\left[T_{0}\left(M^{+}\right)\right]=\mathbb{C}$. 
Proof. (i) Since $R\left[T_{0}\left(M_{j}\right)-\lambda I\right], j=1, \ldots, N$, are proper closed subspaces of $L_{w}^{2}\left(a_{j}, b_{j}\right)$, then the resolvent sets $\rho\left[T_{0}\left(M_{j}\right)\right]$ are empty and hence

$$
\rho\left[T_{0}(M)\right]=\cap_{j=1}^{N} \rho\left[T_{0}\left(M_{j}\right)\right]=\varnothing .
$$

Similarly,

$$
\rho\left[T_{0}\left(M^{+}\right)\right]=\cap_{j=1}^{N} \rho\left[T_{0}\left(M_{j}^{+}\right)\right]=\varnothing .
$$

(ii) Since $R\left[T_{0}\left(M_{j}\right)-\lambda I\right], j=1, \ldots, N$, are closed for any $\lambda \in \mathbb{C}$, then the continuous spectrum of $T_{0}\left(M_{j}\right)$ are the empty sets, that is, $\sigma_{c}\left[T_{0}(M)\right]=\varnothing$, $j=1, \ldots, N$. Hence,

$$
\sigma_{c}\left[T_{0}(M)\right]=\cup_{j=1}^{N} \sigma_{c}\left[T_{0}(M)\right]=\varnothing
$$

Similarly,

$$
\sigma_{c}\left[T_{0}\left(M^{+}\right)\right]=\cup_{j=1}^{N} \sigma_{c}\left[T_{0}\left(M^{+}\right)\right]=\varnothing
$$

(iii) From (i), (ii), and Lemma 3.6, it follows that

$$
\begin{aligned}
\sigma\left[T_{0}(M)\right] & =\cup_{j=1}^{N} \sigma\left[T_{0}\left(M_{j}\right)\right]=\mathbb{C}, \\
\sigma_{r}\left[T_{0}(M)\right] & =\cup_{j=1}^{N} \sigma_{r}\left[T_{0}\left(M_{j}\right)\right]=\mathbb{C} .
\end{aligned}
$$

Similarly,

$$
\begin{aligned}
\sigma\left[T_{0}\left(M^{+}\right)\right] & =\cup_{j=1}^{N} \sigma\left[T_{0}\left(M_{j}^{+}\right)\right]=\mathbb{C}, \\
\sigma_{r}\left[T_{0}\left(M^{+}\right)\right] & =\cup_{j=1}^{N} \sigma_{r}\left[T_{0}\left(M_{j}^{+}\right)\right]=\mathbb{C} .
\end{aligned}
$$

COROLLARY 4.3. Let $T_{0}(M)=\bigoplus_{j=1}^{N} T_{0}\left(M_{j}\right)$ and $T_{0}\left(M^{+}\right)=\bigoplus_{j=1}^{N} T_{0}\left(M_{j}^{+}\right)$, then

(i) $\sigma_{c}[T(M)]=\sigma_{c}\left[T\left(M^{+}\right)\right]=\varnothing$ and $\sigma_{r}[T(M)]=\sigma_{r}\left[T\left(M^{+}\right)\right]=\varnothing$,

(ii) $\sigma[T(M)]=\sigma\left[T\left(M^{+}\right)\right]=\mathbb{C}$ and $\sigma_{p}[T(M)]=\sigma_{p}\left[T\left(M^{+}\right)\right]=\mathbb{C}$,

(iii) $\rho[T(M)]=\rho\left[T\left(M^{+}\right)\right]=\varnothing$.

Proof. From Theorem 3.11 and since $T\left(M_{j}\right)=\left[T_{0}\left(M_{j}^{+}\right)\right]^{*}, j=1, \ldots, N$, it follows that $R\left[T_{0}\left(M_{j}\right)-\lambda I\right], j=1, \ldots, N$, are closed and hence $R[T(M)-\lambda I]=$ $\bigoplus_{j=1}^{N} R\left[T\left(M_{j}\right)-\lambda I\right]$ is closed for every $\lambda \in \mathbb{C}$, see [4, Theorem I.3.7]. Also, by Lemma 3.6, we have

$$
\begin{aligned}
& \operatorname{nul}[T(M)-\lambda I]=\operatorname{def}\left[T_{0}\left(M^{+}\right)-\bar{\lambda} I\right]=\sum_{j=1}^{N} \operatorname{def}\left[T_{0}\left(M_{j}^{+}\right)-\bar{\lambda} I\right]=n N, \\
& \operatorname{def}[T(M)-\lambda I]=\operatorname{nul}\left[T_{0}\left(M^{+}\right)-\bar{\lambda} I\right]=\sum_{j=1}^{N} \operatorname{nul}\left[T_{0}\left(M_{j}^{+}\right)-\bar{\lambda} I\right]=0 .
\end{aligned}
$$


(i) Since $R\left[T\left(M_{j}\right)-\lambda I\right]$ are closed and $\operatorname{def}\left[T\left(M_{j}\right)-\lambda I\right]=0, j=1, \ldots, N$, then, by Lemma 3.6, $R[T(M)-\lambda I]=H$. This yields that $\sigma_{c}[T(M)]=\sigma_{r}[T(M)]=\varnothing$. Similarly,

$$
\sigma_{c}\left[T\left(M^{+}\right)\right]=\sigma_{r}\left[T\left(M^{+}\right)\right]=\varnothing
$$

(ii) Since

$$
\begin{aligned}
\operatorname{nul}[T(M)-\lambda I] & =\sum_{j=1}^{N} \operatorname{nul}\left[T\left(M_{j}\right)-\lambda I\right]=n N, \\
\operatorname{nul}\left[T\left(M^{+}\right)-\bar{\lambda} I\right] & =\sum_{j=1}^{N} \operatorname{nul}\left[T\left(M_{j}^{+}\right)-\bar{\lambda} I\right]=n N, \quad \text { for every } \lambda \in \mathbb{C},
\end{aligned}
$$

then we have

$$
\begin{aligned}
\sigma_{p}[T(M)] & =\cup_{j=1}^{N} \sigma_{p}\left[T\left(M_{j}\right)\right]=\mathbb{C}, \\
\sigma_{p}\left[T\left(M^{+}\right)\right] & =\cup_{j=1}^{N} \sigma_{p}\left[T\left(M_{j}^{+}\right)\right]=\mathbb{C} .
\end{aligned}
$$

It also follows that

$$
\begin{aligned}
\sigma[T(M)] & =\cup_{j=1}^{N} \sigma\left[T\left(M_{j}\right)\right]=\mathbb{C}, \\
\sigma\left[T\left(M^{+}\right)\right] & =\cup_{j=1}^{N} \sigma\left[T\left(M_{j}^{+}\right)\right]=\mathbb{C},
\end{aligned}
$$

and hence,

$$
\rho[T(M)]=\rho\left[T\left(M^{+}\right)\right]=\varnothing .
$$

5. The field of regularity of operators in direct sum spaces. We now obtain some results which, in fact, are a natural consequence of those in Section 4.

THEOREM 5.1. Let $T_{0}(M)=\bigoplus_{p=1}^{N} T_{0}\left(M_{p}\right)$ and $T_{0}\left(M^{+}\right)=\bigoplus_{p=1}^{N} T_{0}\left(M_{p}^{+}\right)$, then

(i) $\Pi\left[T_{0}(M)\right]=\Pi\left[T_{0}\left(M^{+}\right)\right]=\mathbb{C}$ and, for every $\lambda \in \mathbb{C}$,

$$
\operatorname{def}\left[T_{0}(M)-\lambda I\right]=\operatorname{def}\left[T_{0}\left(M^{+}\right)-\bar{\lambda} I\right]=n N,
$$

(ii) $\Pi[T(M)]=\Pi\left[T\left(M^{+}\right)\right]=\mathbb{C}$ and, for every $\lambda \in \mathbb{C}$,

$$
\operatorname{nul}[T(M)-\lambda I]=\operatorname{nul}\left[T\left(M^{+}\right)-\bar{\lambda} I\right]=n N .
$$

Proof. (i) From Theorem 3.11 and Lemma 4.1, for every $\lambda \in \mathbb{C}$, there exists $\left[T_{0}\left(M_{p}\right)-\lambda I\right]^{-1}$ with its domains $R\left[T_{0}\left(M_{p}\right)-\lambda I\right]$ are closed subspaces of $L_{w}^{2}\left(a_{p}, b_{p}\right), p=1, \ldots, N$. Hence, since $T_{0}\left(M_{p}\right), p=1, \ldots, N$, are closed operators, then $\left[T_{0}\left(M_{p}\right)-\lambda I\right]^{-1}$ are also closed; so, it follows from the closed-graph theorem that $\left[T_{0}\left(M_{p}\right)-\lambda I\right]^{-1}, p=1, \ldots, N$, are bounded; hence,

$$
\Pi\left[T_{0}(M)\right]=\cap_{p=1}^{N} \Pi\left[T_{0}\left(M_{p}\right)\right]=\mathbb{C} .
$$


From Theorem 3.11, $R\left[T_{0}\left(M_{p}\right)-\lambda I\right]^{\perp}, p=1, \ldots, N$, are $n$-dimensional subspaces of $L_{w}^{2}\left(a_{p}, b_{p}\right)$. Thus, by Lemma 3.6,

$$
\operatorname{def}\left[T_{0}(M)-\lambda I\right]=\sum_{p=1}^{N} \operatorname{def}\left[T_{0}\left(M_{p}\right)-\lambda I\right]=\sum_{p=1}^{N} \operatorname{dim} R\left[T_{0}\left(M_{p}\right)-\lambda I\right]^{\perp}=n N,
$$

for every $\lambda \in \mathbb{C}$. Similarly,

$$
\begin{aligned}
\operatorname{def}\left[T_{0}\left(M^{+}\right)-\bar{\lambda} I\right] & =\sum_{p=1}^{N} \operatorname{def}\left[T_{0}\left(M_{p}^{+}\right)-\lambda I\right] \\
& =\sum_{p=1}^{N} \operatorname{dim} R\left[T_{0}\left(M_{p}^{+}\right)-\bar{\lambda} I\right]^{\perp}=n N, \quad \text { for every } \lambda \in \mathbb{C} .
\end{aligned}
$$

(ii) As $\Pi\left[T_{0}\left(M^{+}\right)\right]=\mathbb{C}$, for every $\lambda \in \mathbb{C}, T_{0}\left(M^{+}\right)-\bar{\lambda} I$ has closed range; so, since $T(M)=\left[T_{0}\left(M^{+}\right)\right]^{*}, T(M)-\lambda I=\sum_{p=1}^{N}\left[T\left(M_{p}\right)-\lambda I\right]$ has closed range, see [4, Theorem I.3.7]. Furthermore, from (i),

$$
\operatorname{nul}[T(M)-\lambda I]=\operatorname{def}\left[T_{0}\left(M^{+}\right)-\bar{\lambda} I\right]=\sum_{p=1}^{N} \operatorname{def}\left[T_{0}\left(M_{p}^{+}\right)-\bar{\lambda} I\right]=n N .
$$

Hence, $\lambda \notin \Pi[T(M)]$ and so, part (ii) of the theorem follows.

COROLlaRY 5.2. The operators $T_{0}(M)$ and $T_{0}\left(M^{+}\right)$form a compatible adjoint pair with $\Pi\left[T_{0}(M), T_{0}\left(M^{+}\right)\right]=\mathbb{C}$.

Proof. From Theorem 5.1(i) and Lemma 3.7, it follows that

$$
\Pi\left[T_{0}(M), T_{0}\left(M^{+}\right)\right]=\cap_{p=1}^{N} \Pi\left[T_{0}\left(M_{P}\right), T_{0}\left(M_{p}^{+}\right)\right]=\mathbb{C} .
$$

Using (3.19), the corollary follows.

THEOREM 5.3. If, for some $\lambda_{0} \in \mathbb{C}$, there are $n$ linearly independent solutions of the equations

$$
M_{p}\left[\phi_{p}\right]=\lambda_{0} w \phi_{p}, \quad M_{p}^{+}\left[\theta_{p}\right]=\bar{\lambda}_{0} w \theta_{p}, \quad p=1, \ldots, N,
$$

that are in $L_{w}^{2}\left(a_{p}, b_{p}\right)$, then all solutions of the equations

$$
M_{p}\left[\phi_{p}\right]=\lambda w \phi_{p}, \quad M_{p}^{+}\left[\theta_{p}\right]=\bar{\lambda} w \theta_{p}, \quad p=1, \ldots, N,
$$

are in $L_{w}^{2}\left(a_{p}, b_{p}\right)$ for all $\lambda \in \mathbb{C}$.

Proof. The proof follows from Lemmas 3.4 and 3.6, see $[7,13]$ and [14, Lemma 3.3] for more details.

From Corollary 5.2 and Theorem 5.3, we have the following lemma. 
LEMmA 5.4. If, for some $\lambda_{0} \in \mathbb{C}$, there are $n$ linearly independent solutions of the equations

$$
M_{p}\left[\phi_{p}\right]=\lambda_{0} w \phi_{p}, \quad M_{p}^{+}\left[\theta_{p}\right]=\bar{\lambda}_{0} w \theta_{p}, \quad p=1, \ldots, N
$$

that are in $L_{w}^{2}\left(a_{p}, b_{p}\right)$, then $\lambda_{0} \in \Pi\left[T_{0}\left(M_{p}\right), T_{0}\left(M_{p}^{+}\right)\right], p=1, \ldots, N$, see also [17, Theorem 2.1] and [19, Lemma 5.1].

THEOREM 5.5. Let $T_{0}(M)=\bigoplus_{p=1}^{N} T_{0}\left(M_{p}\right)$ and $T_{0}\left(M^{+}\right)=\bigoplus_{p=1}^{N} T_{0}\left(M_{p}^{+}\right)$be the minimal operators defined on the interval $[a, b)$. If $\Pi\left[T_{0}(M), T_{0}\left(M^{+}\right)\right]$is empty, then

$$
\operatorname{def}\left[T_{0}(M)-\lambda I\right]+\operatorname{def}\left[T_{0}\left(M^{+}\right)-\bar{\lambda} I\right] \neq 2 n N .
$$

In particular, if $\Pi\left[T_{0}(M), T_{0}\left(M^{+}\right)\right]$is empty and $n=1$, then

$$
\operatorname{def}\left[T_{0}(M)-\lambda I\right]+\operatorname{def}\left[T_{0}\left(M^{+}\right)-\bar{\lambda} I\right]=N
$$

Proof. If, for some $\lambda_{0} \in \mathbb{C}$, $\operatorname{def}\left[T_{0}(M)-\lambda I\right]=\sum_{p=1}^{N} \operatorname{def}\left[T_{0}\left(M_{p}\right)-\lambda I\right]=n N$ and

$$
\operatorname{def}\left[T_{0}\left(M^{+}\right)-\bar{\lambda} I\right]=\sum_{p=1}^{N} \operatorname{def}\left[T_{0}\left(M_{p}^{+}\right)-\bar{\lambda} I\right]=n N
$$

then each of

$$
M[u]=\lambda_{0} w u, \quad M^{+}[v]=\bar{\lambda}_{0} w v
$$

has $n N-L_{w}^{2}(a, b)$ solutions (see [7]). Hence, by Theorem 5.3, all the solutions of

$$
M[u]=\lambda w u, \quad M^{+}[v]=\bar{\lambda} w v
$$

are in $L_{w}^{2}(a, b)$ for all $\lambda \in \mathbb{C}$; hence, by Corollary 5.2, we have $\lambda \in \Pi\left[T_{0}(M)\right.$, $\left.T_{0}\left(M^{+}\right)\right]$. Thus, if $\Pi\left[T_{0}(M), T_{0}\left(M^{+}\right)\right]$is empty, we cannot have

$$
\operatorname{def}\left[T_{0}(M)-\lambda I\right]+\operatorname{def}\left[T_{0}\left(M^{+}\right)-\bar{\lambda} I\right]=2 n N
$$

In particular, if $n=1$, then, by Lemma 3.4, we have

$$
N \leq \operatorname{def}\left[T_{0}(M)-\lambda I\right]+\operatorname{def}\left[T_{0}\left(M^{+}\right)-\bar{\lambda} I\right] \leq 2 N
$$


so, if $\Pi\left[T_{0}(M), T_{0}\left(M^{+}\right)\right]$is empty, we have

$$
\operatorname{def}\left[T_{0}(M)-\lambda I\right]+\operatorname{def}\left[T_{0}\left(M^{+}\right)-\bar{\lambda} I\right]=N
$$

For a regularly solvable operator, we have the following general theorem.

THEOREM 5.6. Suppose, for a regularly solvable extension $S$ of the minimal operator $T_{0}(M)=\bigoplus_{p=1}^{N} T_{0}\left(M_{p}\right)$, that

$$
\operatorname{def}\left[T_{0}(M)-\lambda I\right]+\operatorname{def}\left[T_{0}\left(M^{+}\right)-\bar{\lambda} I\right]=K, \quad n N \leq K \leq 2 n N,
$$

for all $\lambda \in \Pi\left[T_{0}(M), T_{0}\left(M^{+}\right)\right]$. Then,

$$
\operatorname{nul}[T(M)-\lambda I]+\operatorname{nul}\left[T\left(M^{+}\right)-\bar{\lambda} I\right] \leq K \quad \forall \lambda \in \mathbb{C}
$$

If $\Pi\left[T_{0}(M), T_{0}\left(M^{+}\right)\right]$is empty, then

$$
\operatorname{nul}[T(M)-\lambda I]+\operatorname{nul}\left[T\left(M^{+}\right)-\bar{\lambda} I\right]<K
$$

Proof. Let $\operatorname{def}\left[T_{0}\left(M_{p}\right)-\lambda I\right]=r_{p}$ and $\operatorname{def}\left[T_{0}\left(M_{p}^{+}\right)-\bar{\lambda} I\right]=s_{p}, p=1, \ldots, N$, such that

$$
\operatorname{def}\left[T_{0}\left(M_{p}\right)-\lambda I\right]+\operatorname{def}\left[T_{0}\left(M_{p}^{+}\right)-\bar{\lambda} I\right]=r_{p}+s_{p}, \quad n \leq r_{p}+s_{p} \leq 2 n,
$$

for all $\lambda \in \Pi\left[T_{0}\left(M_{p}\right), T_{0}\left(M_{p}^{+}\right)\right], p=1, \ldots, N$. Then, for any closed extension $S_{p}$ of $T_{0}\left(M_{p}\right)$ which is regularly solvable of $T_{0}\left(M_{p}\right)$ and $T_{0}\left(M_{p}^{+}\right)$, we have, from [4, Theorem III.3.5],

$$
\begin{aligned}
\operatorname{dim}\left\{D\left(S_{p}\right) / D_{0}\left(M_{p}\right)\right\} & =\operatorname{def}\left[T_{0}\left(M_{p}\right)-\lambda I\right]=r_{p}, \quad p=1, \ldots, N \\
\operatorname{dim}\left\{D\left(S_{p}^{*}\right) / D_{0}\left(M_{p}^{+}\right)\right\}=\operatorname{def}\left[T_{0}\left(M_{p}^{+}\right)-\bar{\lambda} I\right]=s_{p}, & p=1, \ldots, N .
\end{aligned}
$$

Hence, $S_{p}$ and $S_{p}^{*}$ are finite-dimensional extensions of $T_{0}\left(M_{p}\right)$ and $T_{0}\left(M_{p}^{+}\right)$, respectively. Thus, from [4, Corollary IX.4.2], we get

$$
\sigma_{e k}\left[T_{0}\left(M_{p}\right)\right]=\sigma_{e k}\left(S_{p}\right) \quad(k=1,2,3 ; p=1, \ldots, N) .
$$

Since $T_{0}\left(M_{p}\right)-\lambda I$ has closed range, zero nullity, and deficiency $r_{p}$ (see Lemma 3.13), then, for any $\lambda \in \mathbb{C}$, we have

$$
\Pi\left[T_{0}\left(M_{p}\right)\right] \cap \sigma_{e k}\left[T_{0}\left(M_{p}^{+}\right)\right]=\varnothing, \quad(k=1,2,3 ; p=1, \ldots, N) .
$$


Therefore,

$$
\triangle_{k}\left[T_{0}\left(M_{p}\right)\right]=\triangle_{k}\left(S_{p}\right)=\mathbb{C} \quad(k=1,2,3 ; p=1, \ldots, N) .
$$

Similarly,

$$
\triangle_{k}\left[T_{0}\left(M_{p}^{+}\right)\right]=\triangle_{k}\left(S_{p}^{*}\right)=\mathbb{C} \quad(k=1,2,3 ; p=1, \ldots, N) .
$$

Furthermore, the equations

$$
M_{p}\left[\phi_{p}\right]=\lambda_{0} w \phi_{p}, \quad M_{p}^{+}\left[\theta_{p}\right]=\bar{\lambda}_{0} w \theta_{p}, \quad p=1, \ldots, N,
$$

have at most $r_{p}$ and $s_{p}$ linearly independent solutions for $\lambda_{0} \in \mathbb{C}$, respectively. Hence,

$$
\begin{aligned}
\operatorname{nul}[ & T(M)-\lambda I]+\operatorname{nul}\left[T\left(M^{+}\right)-\bar{\lambda} I\right] \\
& =\sum_{p=1}^{N} \operatorname{nul}\left[T\left(M_{p}\right)-\lambda I\right]+\sum_{p=1}^{N} \operatorname{nul}\left[T\left(M_{p}^{+}\right)-\bar{\lambda} I\right] \\
& =\sum_{p=1}^{N}\left(r_{p}+s_{p}\right) \leq K, \quad n N \leq K \leq 2 n N \forall \lambda \in \mathbb{C} .
\end{aligned}
$$

But, for any $\lambda_{0} \notin \Pi\left[T_{0}\left(M_{p}\right), T_{0}\left(M_{p}^{+}\right)\right]$, either $\lambda_{0} \notin \Pi\left[T_{0}\left(M_{p}\right)\right]$ or $\bar{\lambda}_{0} \notin$ $\Pi\left[T_{0}\left(M_{p}^{+}\right)\right]$. If $\lambda_{0} \notin \Pi\left[T_{0}\left(M_{p}\right)\right]$, then either $\lambda_{0}$ is an eigenvalue of $T_{0}\left(M_{p}\right)$ or $R\left[T_{0}\left(M_{p}\right)-\lambda I\right], p=1, \ldots, N$, are not closed. Similarly, for $\bar{\lambda}_{0} \notin \Pi\left[T_{0}\left(M_{p}^{+}\right)\right]$. But $T_{0}\left(M_{p}\right)$ and $T_{0}\left(M_{p}^{+}\right)$have no eigenvalues; then, if $\lambda_{0} \notin \Pi\left[T_{0}\left(M_{p}\right), T_{0}\left(M_{p}^{+}\right)\right]$, we have $R\left[T_{0}\left(M_{p}\right)-\lambda I\right]$ and $R\left[T_{0}\left(M_{p}^{+}\right)-\bar{\lambda} I\right], p=1, \ldots, N$, are both not closed, and so we cannot have

$$
\begin{aligned}
\operatorname{nul} & {[T(M)-\lambda I]+\operatorname{nul}\left[T\left(M^{+}\right)-\bar{\lambda} I\right] } \\
& =\sum_{p=1}^{N} \operatorname{nul}\left[T\left(M_{p}\right)-\lambda I\right]+\sum_{p=1}^{N} \operatorname{nul}\left[T\left(M_{p}^{+}\right)-\bar{\lambda} I\right]=K .
\end{aligned}
$$

Hence,

$$
\operatorname{nul}[T(M)-\lambda I]+\operatorname{nul}\left[T\left(M^{+}\right)-\bar{\lambda} I\right]<K, \quad n N \leq K \leq 2 n N,
$$

for all $\lambda \notin \Pi\left[T_{0}(M), T_{0}\left(M^{+}\right)\right]=\cap_{p=1}^{N} \Pi\left[T_{0}\left(M_{p}\right), T_{0}\left(M_{p}^{+}\right)\right]$.

REMARK 5.7. It remains an open question as to how many of the solutions of the equations

$$
M[u]=\lambda w u, \quad M^{+}[v]=\bar{\lambda} w v
$$

may be in $L_{w}^{2}(a, b)$ for any $\lambda \in \mathbb{C}$, when $\Pi\left[T_{0}(M), T_{0}\left(M^{+}\right)\right]$is empty, except that we know from above that not all of them are in $L_{w}^{2}(a, b)$. We refer to $[3,7,17,19]$ for more details. 


\section{REFERENCES}

[1] N. I. Akhiezer and I. M. Glazman, Theory of Linear Operators in Hilbert Space. Vol. I, Frederick Ungar Publishing, New York, 1961.

[2] _ , Theory of Linear Operators in Hilbert Space. Vol. II, Frederick Ungar Publishing, New York, 1963.

[3] J. Chaudhuri and W. N. Everitt, On the spectrum of ordinary second order differential operators, Proc. Roy. Soc. Edinburgh Sect. A 68 (1969), 95-119.

[4] D. E. Edmunds and W. D. Evans, Spectral Theory and Differential Operators, Oxford Mathematical Monographs, Oxford University Press, New York, 1987.

[5] W. D. Evans, Regularly solvable extensions of nonselfadjoint ordinary differential operators, Proc. Roy. Soc. Edinburgh Sect. A 97 (1984), 79-95.

[6] W. D. Evans and S. E. Ibrahim, Boundary conditions for general ordinary differential operators and their adjoints, Proc. Roy. Soc. Edinburgh Sect. A 114 (1990), no. 1-2, 99-117.

[7] W. N. Everitt, Integrable-square solutions of ordinary differential equations. III, Quart. J. Math. Oxford Ser. (2) 14 (1963), 170-180.

[8] W. N. Everitt and D. Race, Some remarks on linear ordinary quasidifferential expressions, Proc. London Math. Soc. (3) 54 (1987), no. 2, 300-320.

[9] W. N. Everitt and A. Zettl, Generalized symmetric ordinary differential expressions. I. The general theory, Nieuw Arch. Wisk. (3) 27 (1979), no. 3, 363397.

[10] _ Sturm-Liouville differential operators in direct sum spaces, Rocky Mountain J. Math. 16 (1986), no. 3, 497-516.

[11] _ Differential operators generated by a countable number of quasidifferential expressions on the real line, Proc. London Math. Soc. (3) 64 (1992), no. 3, 524-544.

[12] S. E. Ibrahim, The point spectra and regularity fields of non-self-adjoint quasidifferential operators, Rocky Mountain J. Math. 25 (1995), no. 2, 685-699.

[13] _ The spectra of well-posed operators, Proc. Roy. Soc. Edinburgh Sect. A 125 (1995), no. 6, 1331-1348.

[14] _ On the essential spectra of regularly solvable operators in the direct sum spaces, Rocky Mountain J. Math. 29 (1999), no. 2, 609-644.

[15] M. A. Naimark, Linear Differential Operators. Part I: Elementary Theory of Linear Differential Operators, Frederick Ungar Publishing, New York, 1967.

[16] _ Linear Differential Operators. Part II: Linear Differential Operators in Hilbert Space, Frederick Ungar Publishing, New York, 1968.

[17] D. Race, On the location of the essential spectra and regularity fields of complex Sturm-Liouville operators, Proc. Roy. Soc. Edinburgh Sect. A 85 (1980), no. 1-2, 1-14.

[18] _ On the essential spectra of linear 2 nth order differential operators with complex coefficients, Proc. Roy. Soc. Edinburgh Sect. A 92 (1982), no. 1-2, 65-75.

[19] _ The theory of J-selfadjoint extensions of J-symmetric operators, J. Differential Equations 57 (1985), no. 2, 258-274.

[20] M. I. Visik, On general boundary problems for elliptic differential operators, Amer. Math. Soc. Transl. (2) 24 (1963), 107-172.

[21] A. Zettl, Formally self-adjoint quasi-differential operators, Rocky Mountain J. Math. 5 (1975), no. 3, 453-474.

Sobhy El-Sayed Ibrahim: Department of Mathematics, Faculty of Science, Benha University, Benha 13518, Kalubia, Egypt 


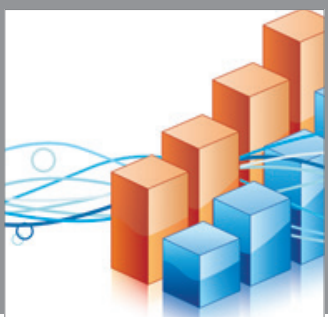

Advances in

Operations Research

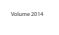

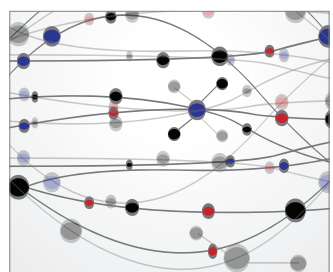

\section{The Scientific} World Journal
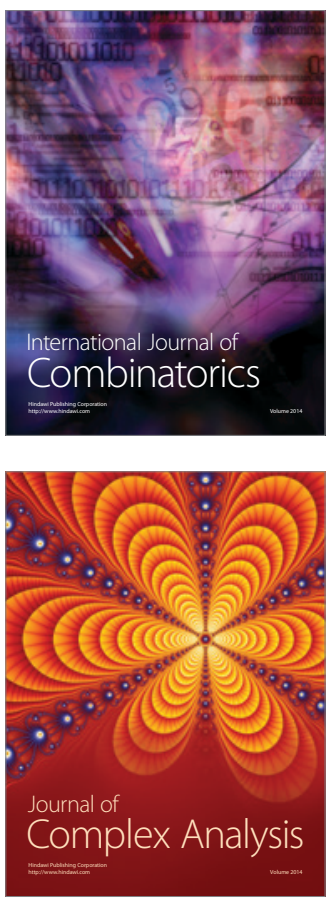

International Journal of

Mathematics and

Mathematical

Sciences
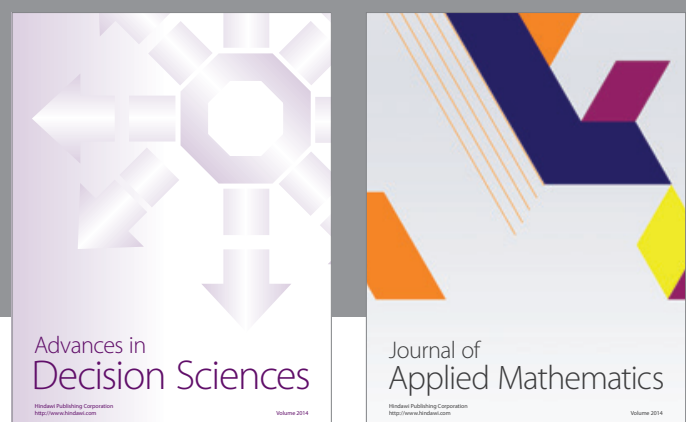

Journal of

Applied Mathematics
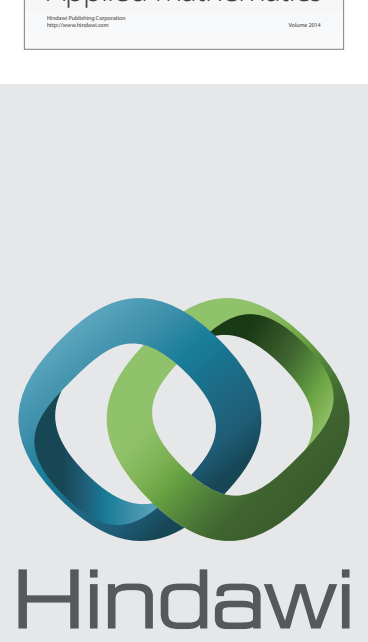

Submit your manuscripts at http://www.hindawi.com
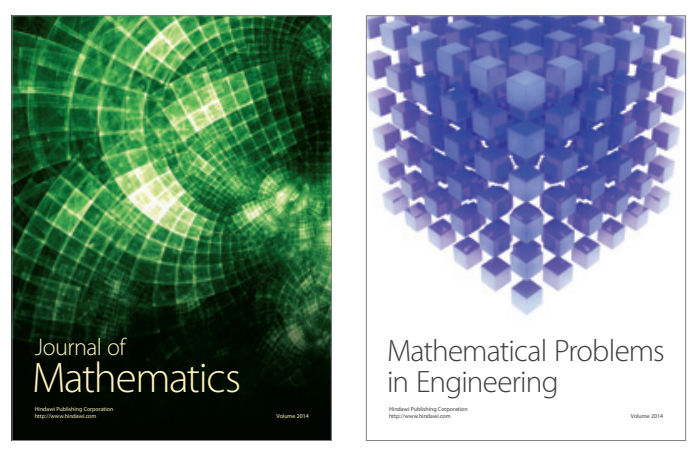

Mathematical Problems in Engineering
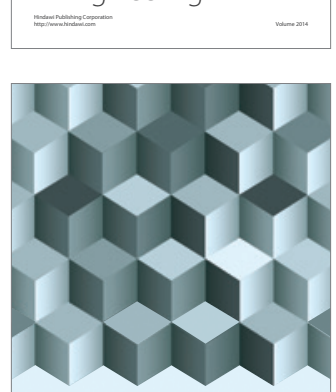

Journal of

Function Spaces
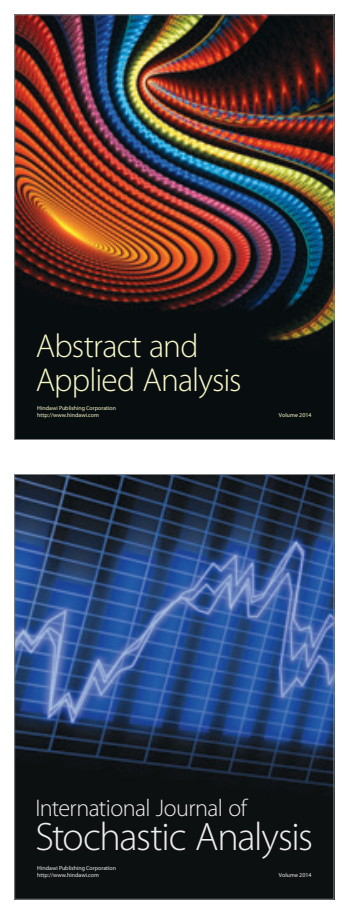

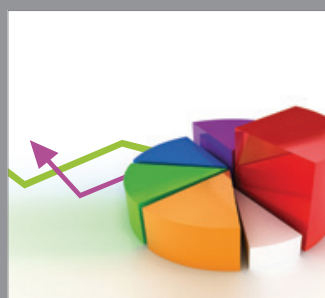

ournal of

Probability and Statistics

Promensencen
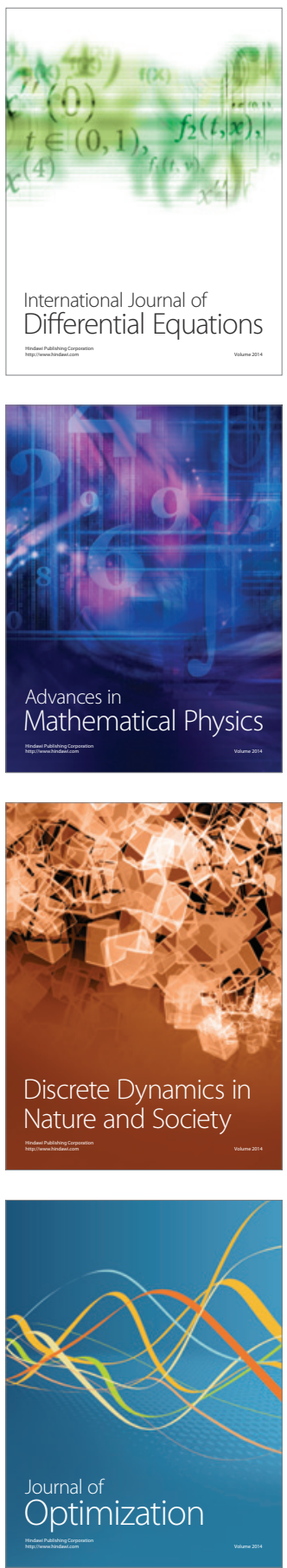